\title{
IncRNA-RMST Functioned as a SOX2 Transcription Co-Regulator to Regulate miR-1251 in the Progression of Hirschsprung's Disease
}

\section{Lingling Zhou}

Soochow University Affiliated Children's Hospital

\section{Zhengke Zhi}

Children's Hospital of Nanjing Medical University

\section{Pingfa Chen}

Yancheng city NO.1 People's Hospital

Chunxia Du

Children's Hospital of Nanjing Medical University

\section{Binyu Wang}

Children's Hospital of Nanjing Medical University

\section{Xiang Fang}

Children's Hospital of Nanjing Medical University

\section{Weibing Tang}

Children's Hospital of Nanjing Medical University

Hongxing Li ( $\square$ hx8817@njmu.edu.cn )

Children's hospital of Nanjing Medical University https://orcid.org/0000-0002-8178-4622

\section{Research}

Keywords: Hirschsprung's disease, IncRNA-RMST, miR-1251, SOX2

Posted Date: January 18th, 2021

DOI: https://doi.org/10.21203/rs.3.rs-147665/v1

License: (c) (1) This work is licensed under a Creative Commons Attribution 4.0 International License. Read Full License 


\section{Abstract}

\section{Background}

Hirschsprung's disease (HSCR) is a congenital colon disease characterized by the lack of ganglion cells which is closely related to impaired migration and proliferation of enteric neural crest cells (ENCCs). Long non-coding RNAs (IncRNAs) and microRNAs (miRNAs) have been authenticated as an important class of regulators of biological functions. In this study, a microarray analysis was conducted and found that Rhabdomyosarcoma 2-associated transcript (RMST), a newly defined IncRNA, was down-regulated in the stenotic segment of HSCR patients. MiR-1251 is transcribed from the intron region of RMST and was also low expressed in the aganglionic tract. This study aimed to clarify the roles they played in the occurrence of HSCR.

Methods

QRT-PCR was applied to validate the mRNA expression of RMST, miR-1251, SOX2 and AHNAK, while western blotting was employed to evaluate the protein level of SOX2 and AHNAK in clinical samples and cells. CCK-8 and transwell assays were used to detect cell proliferation and migration after transfection. Chromatin immunoprecipitation (CHIP) was applied to confirm SOX2 could bind to the promoter region of miR-1251. RNA binding protein immunoprecipitation (RIP) was used to demonstrate the interaction between RMST and SOX2. Dul-luciferase reporter assay was performed to confirm miR-1251 could interact with AHNAK.

Results

When the expression of RMST or miR-1251 was reduced, cell proliferation and migration was attenuated. However, RMST not to influence the expression of miR-1251 directly. Through bioinformatic analysis, transcription factor SOX2 was predicted to bind to the promoter region of miR-1251 which was confirmed by CHIP assay. We also demonstrated that RMST exerted as a co-regulator of SOX2 via RIP assay. AHNAK was predicted as the downstream gene of miR-1251 confirmed by the dual-luciferase reporter assay. Furtherly, rescue experiments showed RMST functioned as a transcription co-regulator of SOX2 to upregulate the expression of downstream gene AHNAK by strengthening the regulation of SOX2 on miR1251 in HSCR.

Conclusions

These findings uncovered the role of RMST/SOX2/miR-1251/AHNAK pathway during the development of Hirschsprung's disease and presented potential therapeutic targets for HSCR.

\section{Background}

Hirschsprung disease (HSCR), a common enteric neuropathy, is characterized by the absence of gangliocytes in the distal colon[1, 2]. During 5 to 12 weeks of embryogenesis, enteric neural crest cells 
(ENCCs) failed to migrate and proliferate might cause this disease [3]. HSCR usually attacks about $1 / 5000$ neonates, while the incidence rate of females is about a quarter of males. [4]. Current etiological studies show that HSCR is a complicated disorder involving multiples genetic factors[5]. Genes including RET, GDNF, GFRA1, EDNRB and PHOX2B have been confirmed to be involved in HSCR [6, 7]. However, these genes could only explain partly, so further research is needed.

With longer than 200 nucleotides, long noncoding RNAs (IncRNAs) are increasingly considered to be the main players in governing basic biological processes by affecting gene expression at nearly all levels.[8, 9]. As reported before, various IncRNAs can regulate cell proliferation and migration. For instance, IncRNA TPTEP1 could inhibit the non-small cell lung cancer (NSCLC) cells to proliferate through abating miR-328-5p expression[10]. In addition, in renal cell carcinoma, IncRNA00312 attenuated cell proliferation and migration obviously[11]. However, the study about IncRNA functioned in HSCR is rarely reported.

In order to explore the role of IncRNA in the occurrence of HSCR, a microarray was conducted in this study, and we found IncRNA Rhabdomyosarcoma 2-Associated Transcript (RMST) was significantly low expressed in the aganglionic bowels compared with the normal ones. RMST has been discovered to be essential in neuronal differentiation[12,13]. According to a report, RMST promoted activation of microglial cells by activating TAK1-mediated NF-KB signaling[14]. Considering the effects of RMST on nervous system and the low-expressed of RMST in HSCR, we aimed to reveal its roles during the procedure of HSCR. Furtherly, miR-1251 was transcribed from the same genomic site as RMST and was also low-expressed in HSCR diseased bowel. However, we found RMST didn't regulate its intron gene miR1251 independently in this study. There may be other regulatory mechanisms to be explored.

Sex determining region $\mathrm{Y}$ (SRY)-box 2 (SOX2) functions a transcription factor is implicated in transcriptional regulation[15, 16]. It has also been discovered to regulate miRNAs expression[17]. Interestingly, SOX2 is closely related to the nervous system, such as the terminal differentiation of postmitotic olfactory neurons was regulated by SOX2 directly [18]. Through bioinformatics analysis, it was found SOX2 might bind to the promoter of miR-1251. Numerous evidences have also indicated that the down-regulation of SOX2 attenuated cell growth and migration $[19,20]$. Thus, SOX2 probably be related to the development of neural crest cells during HSCR by regulating the expression of miR-1251.

Furthermore, according to previous report, RMST could interact with SOX2 and then enhance its regulation on downstream genes[21]. Therefore, we hypothesized that RMST might function as a SOX2 transcription co-regulator to regulate the downstream miR-1251 and participate in the proceeding of HSCR.

\section{Methods}

\section{Clinical information}

This study was approved by the Institutional Ethics Committee of Nanjing Medical University (NJMU Birth Cohort), and the experiments were carried out according to approved guidelines. 32 stenotic colon tissues 
were collected from patients accepted radical operation of HSCR in Children's Hospital of Nanjing Medical University from January 2011 to August 2014. 32 controls matched with cases on age and gender were randomly picked out from isolated patients on account of intussusception or incarcerated and strangulated inguinal hernia without the ischemia or necrosis parts. Tissues were harvested and stored at $-80^{\circ} \mathrm{C}$ immediately after surgery. All HSCR patients were diagnosed through pathological analysis. Finally, written informed consent from all participants were obtained.

\section{Quantitative Real-time Polymerase Chain Reaction (qRT-PCR)}

To isolate total RNA from tissues and cells, Trizol reagent (Invitrogen Life Technologies Co, USA) was applied. QRT-PCR was employed to detect RMST, miR-1251 and AHNAK expression. TaqMan® MicroRNA Assays (Applied Biosystems, USA) was used to test miR-1251 expression. GAPDH and U6 was applied as an internal control for mRNA and miRNA detection, respectively. Roche LightCycler480 (Roche, Switzerland) was used to perform qRT-PCR depending on the manufacturer's protocol. Primer sequences were showed in Supplement Table 1.

\section{Western Blotting}

RIPA lysis buffer (Beyotime, Shanghai, China) was applied to extract total proteins from colon tissues and cultured cells. BCA Protein Assay Kit (Beyotime, Shanghai, China) was used to detect protein concentration. The same amount of total proteins was isolated in 10\% SDS-PAGE, transferred to PVDF membranes and then sealed for $1 \mathrm{~h}$. At $4^{\circ} \mathrm{C}$, primary antibodies were used for incubation overnight. Afterwards, corresponding secondary antibodies were added for $2 \mathrm{~h}$ at $25^{\circ} \mathrm{C}$. Finally, the membranes were exposed via ECL and Western blot detection reagents (Thermo Fisher Scientific, MA, USA). Antibodies including anti-AHNAK (SC134252), anti-SOX2 (SC17320X) and anti-GAPDH (SC47724) were obtained from Santa Cruz (CA, USA). The corresponding secondary antibodies were obtained from Beyotime (Shanghai, China).

\section{Chromatin Immunoprecipitation (ChIP)}

By using ChIP Assay Kit (Thermo Fisher Scientific, Shanghai, China), ChIP was implemented in accordance with the operating instructions. Firstly, cross-linked chromatin was sonicated into around 200 bp to $1000 \mathrm{bp}$ fragments. Anti-SOX2 was used to immunoprecipitate the chromatin. Goat immunoglobulin G (IgG, ab172730) was applied to be the negative control. PCR was performed using SYBR Green Mix (Takara Bio, Japan). The primer sequences were shown in Supplement Table 1.

\section{Cell culture and transfection}

SH-SY5Y and 293T cell lines were acquired from ATCC. Cells were cultured at $37^{\circ} \mathrm{C}, 5 \% \mathrm{CO}_{2}$ condition using DMEM (Hyclone, USA) culture medium containing 10\% FBS, $100 \mathrm{U} / \mathrm{mL}$ penicillin, and $100 \mu \mathrm{g} / \mathrm{mL}$ streptomycin. The inhibitor of miR-1251, siRNA of RMST, SOX2 and the corresponding negative controls 
were synthesized by Genechem (Shanghai, China). Transfection experiments were conducted by using Lipofectamine 2000 Reagent (Invitrogen, USA).

\section{Cell proliferation assay}

To test the cell viability, cell counting kit-8 (CCK-8, Dojindo, Japan) was employed. After transfection, cells were cultured in 96-well plates for 24-48 $\mathrm{h}$ and then cells were incubated with CCK-8 reagent the for 1-2 $\mathrm{h}$. Eventually, the OD value at $450 \mathrm{~nm}$ was detected by the TECAN infinite M200 Multimode microplate reader (Tecan, Mechelen, Belgium). Each assay was conducted independently in triplicate.

\section{Cell migration assay}

Transwell chambers were placed above a 24-well plate. After transfection around $24-48 \mathrm{~h}$, cells were resuspended with serum-free medium to $1 \times 10^{6} \mathrm{cell} / \mathrm{s} / \mathrm{ml}$. About $100 \mu \mathrm{l}$ cell suspension was seeded to the upper chamber. $500 \mu \mathrm{L}$ of complete culture medium containing FBS was added to the lower chamber. 24$48 \mathrm{~h}$ later, $4 \%$ paraformaldehyde was applied to fix the lower chamber cells and then crystal violet staining solution was used to stain cells. Cells migrated to the lower chamber were counted and imaged using an inverted microscope $(\times 20)$. All experiments were conducted in triplicate.

\section{Dual-luciferase reporter assay}

The predicted 3'-UTR sequence of AHNAK binding to miR-1251 and the mutated sequence were inserted into the pGL3 promoter vector (Genechem, Shanghai, China) named pGL3-AHNAK-WT and pGL3-AHNAKMUT. For reporter assay, cells were planted into 24-well plates and transfected with 100ng of pGL3AHNAK-WT and pGL3-AHNAK-MUT, 50nM miR-1251 mimics and negative control using Lipofectamine 2000. Renilla luciferase vector pRL-SV40 (5 ng) was transfected into cells as control. Based on the obtained ratio, the activation degree of target reporter genes in different sample was compared.

\section{Statistical analysis}

GraphPad Prism 7.0 (GraphPad Software, USA) was adopted to analyze data. Between two groups, $t$-test was applied to determine the statistically significant differences, while the comparison among multiple groups was performed via one-way ANOVA. All data were presented as the mean \pm SEM. $P<0.05$ was considered to be statistically significant.

\section{Results}

\section{Down-regulation of RMST and miR-1251 in HSCR patients}

To verify the expression levels of RMST, qRT-PCR was employed. RMST was markedly down-regulated in ganglia-free intestinal segment compared with normal controls as result showed (Fig. 1a). In order to detect whether RMST could affect cell migration and proliferation, Transwell and CCK8 assays were conducted. As the results showed, after transfected with RMST siRNA, the migrated and proliferated cells 
were obviously fewer than the normal control in both cell lines. (Fig. 1b). We also discovered the RMST intronic miR-1251 was down-regulated in aganglionic tracts (Fig. 1c). When cells transfected with miR1251 inhibitor, the cell migration and proliferation was attenuated (Fig. 1d).

\section{miR-1251 was transcriptionally regulated by SOX2}

Because miR-1251 is transcribed from the same genomic locus as RMST, we suspected that RMST might influence the expression level of miR-1251. To verify it, we knocked down RMST in SH-SH-SY5Y and 293T cells and then measured miR-1251 levels by qRT-PCR, however, there was no significant changes on miR1251 expression level in both cell lines, indicating that RMST was not a precursor transcript for it (Supplement Figure). Furtherly, we employed bioinformatics approach Promoter Scan (http://www.ncbi.nlm.nih.gov/Class/NAWBIS/Modules/DNA/dna21b.html) to predict the transcription promoter of miR-1251. SOX2 was predicted to bind with the 2kbp upstream promoter region of miR-1251 (Supplement Figure). To confirm the combinative relationship between SOX2 and miR-1251, ChIP experiment was performed in the 293T cells. The result confirmed that SOX2 could bind to the promoter region of miR-1251 (Fig. 2a). Furtherly, when we abated the level of SOX2, miR-1251 was down-regulated obviously (Fig. 2b). Additionally, SOX2 was found down-regulated at mRNA and protein levels in HSCR patients than normal controls. (Fig. 2c, d). Based on this, we supposed that SOX2 influenced cell migration and proliferation by regulating miR-1251. As expected, when cells were transfected with SOX2 siRNA, cell proliferation and migration was attenuated, while upregulating miR-1251 could reverse it partly (Fig. 2e-g).

\section{RMST functioned as a co-regulator of SOX2}

As reported before, RMST could also combine with SOX2 and then enhanced the transcriptional function of SOX2. In this study, we demonstrated that RMST could bind with SOX2 using RIP assay (Fig. 3a). Therefore, we forecasted that miR-1251 was transcriptionally regulated by SOX2 and RMST could strengthen this effect. When we knocked down the expression of SOX2 and both SOX2 and RMST in $293 T$ cell, respectively, the expression of miR-1251 was detected. As expected, miR-1251 was downregulated after cells transfected with SOX2 siRNA and was much lower in cells co-transfected with RMST siRNA and SOX2 siRNA (Fig. 3b). So, whether RMST exerted its roles through SOX2/miR-1251 axis? Through CCK-8 and Tranwell assays, we found that when the expression of RMST and SOX2 were both knocked down, the cell proliferation and migration were more weakened than just down-regulated RMST or SOX2 alone. Meanwhile, the upregulation of miR-1251 partly reversed the co-function of si-RMST and si-SOX2 (Fig. 3c, d). These results revealed that RMST might function via acting as a co-regulator of SOX2 to regulate downstream gene miR-1251.

\section{AHNAK was the target gene of miR-1251.}

To predict the downstream target gene of miR-1251, bioinformatical analysis was employed and we found miR-1251 might bind to the 3'UTR of AHNAK (Fig. 4a). As dul-luciferase reporter assay showed, compared with the control group, the luciferase activity was significantly decreased when cells co- 
transfected with miR-1251 mimics and pGL3-AHNAK-WT plasmids demonstrating the relationship between miR-1251 and AHNAK (Fig. 4b). Furtherly, miR-1251 inhibitor was transfected in 293T cells. After 24 hours, AHNAK mRNA and protein levels were found up-regulated in 293T cells (Fig. 4c, d). By qRT-PCR, AHNAK was demonstrated up-regulated remarkably in aganglionic tract compared with normal controls (Fig. 4e). The protein level of AHNAK was detected furtherly, and was fit with its mRNA expression level (Fig. 4f). Meanwhile, we explored whether miR-1251 regulated cell function through AHNAK. As rescue experiment results showed, the reduction of AHNAK could partly reverse the influence of miR-1251 inhibitor on both cell migration and proliferation (Fig. 4g-i).

\section{RMST played as a SOX2 transcription co-regulator to inhibit miR-1251 and raise AHNAK expression}

Combined with above results, we presumed that RMST enhanced the regulation of SOX 2 to miR-1251 and then promoted the expression of AHNAK, finally खaffecting the proliferation and migration of neural

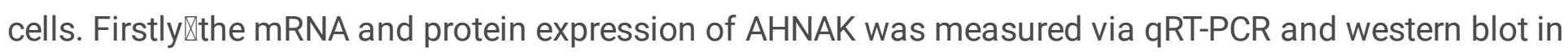
every group respectively. There was no significant difference found in AHNAK expression between the RMST low expression group and the control group, however, the mRNA and protein expression of AHNAK was increased after SOX2 was down regulated. Furthermore, the expression of AHNAK was much lower in "RMST siRNA+SOX2 siRNA" group than in "SOX2 siRNA" group. which confirmed that RMST, as a SOX2 transcription co regulatory factor, upregulated the expression of AHNAK (Fig. 5a, b). In order to further confirmed the mechanism of its function through AHNAK, RMST low expression group, SOX2 low expression group, RMST low expression + SOX2 low expression group, RMST low expression + SOX2 low expression + AHNAK low expression group and control group were set up. CCK-8 and Transwell assays were applied to detect cell proliferation and migration abilities of every group. The results showed that the combined inhibition of both RMST and SOX2 low expression on cell proliferation and migration could be partially alleviated by simultaneously downregulating the expression of AHNAK (Fig. 5c, d).

\section{Discussion}

Non coding RNA (ncRNA) has been once considered as the "noise" of human genome transcription, which has no biological effects. With the development of high-throughput sequencing technology and in-depth research, more and more ncRNAs, especially miRNA and IncRNA, have been found to play very important roles in epigenetic regulation and take part in the occurrence and development of multiple diseases[2224]. Although there have been some reports about ncRNAs in HSCR, its mode of action and mechanism still need further study[25, 26]. In this study, our research team detected that in the stenosis tract of HSCR patients, RMST and miR-1251 were down-regulated apparently. MiR-1251 was firstly demonstrated as a potential prognostic markers in head and neck squamous cell carcinoma[27]. But there're few studies about miR-1251 in other diseases. Herein, it was initially found that the cell proliferation and migration was significantly inhibited after the expression of RMST or miR-1251 was reduced, indicating that RMST and miR-1251 might play a certain role in the pathogenesis of HSCR. Although miR-1251 derived from the intron region of RMST, we found RMST did not regulate the expression of miR-1251 independently. Whether there's other regulatory mechanism? We explored it in the next step. 
According to the bioinformatical analysis, we found SOX2, a transcription factor, probably bind to the promoter region of miR-1251. ChIP assay then confirmed it. SOX family, such as SOX10 has been proved to be related to the pathogenesis of HSCR[28], but there are few reports about the role and mechanism of SOX2 in the occurrence of HSCR. As reported before, SOX2 could regulate the proliferation and differentiation of peripheral nerve cells in the peripheral nervous system, [29]. A recent study revealed that SOX2 gene was involved in the development of embryonic neural tube and neural crest cells[30]. When knocked out SOX2 gene, the number of neurons in the ganglion derived from neural crest of mouse embryo decreased obviously, indicating SOX2 probably also play an important role in the progression of HSCR [31]. Furtherly, SOX2 could bind to bivalently marked promoters of poised pro-neural and neurogenic genes, and then activated neuronal differentiation appropriately [32]. These findings all indicated the important role of SOX2 played in the nervous system. In addition, over-expressed SOX2 promoted tumor progression by enhancing the abilities of cell proliferation and migration[17, 33]. Herein, we demonstrated SOX2 was significantly down-regulated in aganglionic tract and the following experiments showed the lowly expressed SOX2 might be involved in the occurrence of HSCR by repressing neural crest cells' proliferation and migration via regulating miR-1251.

LncRNAs could bind with some proteins to influence their function. For example, IncRNA EPIC1 accelerated the regulation of MYC on downstream genes through combing with MYC[34]. In this study, RIP results showed that RMST and SOX2 had binding relationship, which provided a basis for RMST as a transcription co-regulator of SOX2 to regulate miR-1251. We also found that the inhibition on cell proliferation and migration was more obvious in knocked down both RMST and SOX2 than abated RMST or SOX2 expression alone. Furtherly, raised miR-1251 partially alleviated the combined effect of RMST and SOX2 which confirmed that RMST might play a role as a transcription co-regulatory factor of SOX2 by enhancing the regulation of SOX2 on miR-1251. Previous studies about IncRNAs in HSCR have mostly focused on the mechanism of competitive endogenous RNA (ceRNA) $[35,36]$. This research, however, firstly explored the mechanism of HSCR from the perspective of IncRNA binding protein, and expanded a new direction of HSCR research.

MiRNAs generally perform their functions by degrading their target genes[37]. It was confirmed in this study that AHNAK was the target gene of miR-1251 through the analysis of biological information and the experiment of dul-luciferase reporter assay. AHNAK, as a kind of scaffold protein, is involved in the regulation of $\mathrm{Ca}_{2}{ }^{+}$channel and the formation of actin cytoskeleton, which has a profound impact on cell migration function[38]. For example, it has been found that AHNAK is significantly under-expressed in breast cancer and in vitro experimental results show that the proliferation and migration ability of cells is significantly impaired after the up-regulation of AHNAK[39]. Owing to the expression level of AHNAK in the stenosis tract of HSCR was significantly higher than control ones, whether miR-1251 functioned through AHNAK was investigated in this study. As expected, miR-1251 inhibited cell proliferation and migration, but improved AHNAK expression could partly reverse it.

Thus $₫$ whether RMST, as a co transcription regulator of SOX2, up-regulated the expression of AHNAK by regulating miR-1251 and exerted its roles through RMST/SOX2/miR-1251/AHNKA axis needed deeply 
study. As results showed, none significant difference of AHNAK expression between si-RMST group and control group was found, which also confirmed that RMST did not regulate miR-1251 alone. But the expression of AHNAK improved more a lot when co-reduced RMST and SOX2 than only down-regulating SOX2. Meanwhile, the results of cell function experiments revealed that the down-regulation of AHNAK partially reversed the inhibition of RMST and SOX2 simultaneous down-regulation on cell proliferation and migration, indicating that RMST, as a co-transcription regulator of SOX2, could affect the expression of downstream gene AHNAK through miR-1251 and then influence neural cells' migration and proliferation.

\section{Conclusion}

This study revealed that RMST functioned as a co-transcription regulator of SOX2 to upregulate the expression of downstream gene AHNAK by strengthening the regulation of SOX2 on miR-1251 for the first time, which probably be involved in the pathogenesis of HSCR. The discovery of RMST/SOX2/miR1251/AHNAK could be helpful for the targeted therapy of HSCR in the future.

However, this study. still existed some deficiencies We discovered that decreased the expression of RMST alone could also inhibit cell proliferation and migration, whether RMST has other regulation pattern needs further study. In addition, due to the animal model of HSCR is hard to be established, this study is not supported by in vivo experiments, though we're trying to overcome this shortage to make our study more persuasive.

\section{Abbreviations}

HSCR: Hirschsprung's disease; ENCCs: enteric neural crest cells; IncRNAs: long non-coding RNAs; miRNAs: microRNAs; RMST: Rhabdomyosarcoma 2-associated transcript; SOX2: Sex determining region Y (SRY)-box 2; CHIP: chromatin immunoprecipitation; RIP: RNA binding protein immunoprecipitation;

\section{Declarations}

\section{Ethics approval and consent to participate}

This study was approved by the Institutional Ethics Committee of Nanjing Medical University (NJMU Birth Cohort), and the experiments were conducted in accordance with the principles of the Declaration of Helsinki. All parents of patients had provided written informed consent in the study.

\section{Consent for publication}

This article does not contain any studies with human participants or animals performed by any of the authors. 
The datasets used and/or analyzed during the current study are available

from the corresponding author on reasonable request.

\section{Competing interests}

The authors declare that they have no competing interests.

\section{Funding}

This work was supported by the Natural Science Foundation of China (NSFC 81701493) and General project of Nanjing health and Family Planning Commission (YKK19103).

\section{Authors' contribution}

Designed the project: Hongxing Li; Performed the experiments: Lingling Zhou, Zhengke Zhi, Pingfa Chen; Analyzed the data: Lingling Zhou, Wrote the paper: Zhengke Zhi. All authors discussed the results and commented on the manuscript.

\section{Acknowledgements}

We thank Dr. Jie Zhang, Xiaofeng Lv, Weiwei Jiang, Huan Chen, Wei Li, Changgui Lu (Children's Hospital of Nanjing Medical University) for sample collection. This work was supported by the Natural Science Foundation of China (NSFC 81701493).

\section{References}

1. Sergi C: Hirschsprung's disease: Historical notes and pathological diagnosis on the occasion of the 100 (th) anniversary of Dr. Harald Hirschsprung's death. World J Clin Pediatr 2015, 4:120-125.

2. Jaroy EG, Acosta-Jimenez L, Hotta R, Goldstein AM, Emblem R, Klungland A, Ougland R: "Too much guts and not enough brains": (epi)genetic mechanisms and future therapies of Hirschsprung disease - a review.Clin Epigenetics 2019, 11:135.

3. Bergeron KF, Silversides DW, Pilon N: The developmental genetics of Hirschsprung's disease.Clin Genet 2013, 83:15-22.

4. Wester T, Granstrom AL: Hirschsprung disease-Bowel function beyond childhood.Semin Pediatr Surg 2017, 26:322-327.

5. McKeown SJ, Stamp L, Hao MM, Young HM: Hirschsprung disease: a developmental disorder of the enteric nervous system. Wiley Interdiscip Rev Dev Biol 2013, 2:113-129.

6. Tam PK: Hirschsprung's disease: A bridge for science and surgery.J Pediatr Surg 2016, 51:18-22.

7. Zhao J, Zhu Y, Xie X, Yao Y, Zhang J, Zhang R, Huang L, Cheng J, Xia H, He J, Zhang Y: Pleiotropic effect of common PHOX2B variants in Hirschsprung disease and neuroblastoma.Aging (Albany NY) 2019, 11:1252-1261. 
8. Xu Z, Liu C, Zhao Q, Lu J, Ding X, Luo A, He J, Wang G, Li Y, Cai Z, et al: Long non-coding RNA CCAT2 promotes oncogenesis in triple-negative breast cancer by regulating stemness of cancer cells.Pharmacol Res 2020, 152:104628.

9. Shen S, Wang J, Zheng B, Tao Y, Li M, Wang Y, Ni X, Suo T, Liu H, Liu H, Zhang J: LINC01714 Enhances Gemcitabine Sensitivity by Modulating FOX03 Phosphorylation in Cholangiocarcinoma. Mol Ther Nucleic Acids 2019, 19:446-457.

10. Cao F, Wang Z, Feng Y, Zhu H, Yang M, Zhang S, Wang X: IncRNA TPTEP1 competitively sponges miR3285p to inhibit the proliferation of nonsmall cell lung cancer cells. Oncol Rep 2020.

11. Zeng J, Li Y, Wang Y, Xie G, Feng Q, Yang Y, Feng J: IncRNA 00312 Attenuates Cell Proliferation and Invasion and Promotes Apoptosis in Renal Cell Carcinoma via miR-34a-5p/ASS1 Axis. Oxid Med Cell Longev 2020, 2020:5737289.

12. Cheng H, Sun M, Wang ZL, Wu Q, Yao J, Ren G, Sun XL: LncRNA RMST-mediated miR-107 transcription promotes OGD-induced neuronal apoptosis via interacting with hnRNPK.Neurochem Int 2020, 133:104644.

13. Tang W, Tang J, He J, Zhou Z, Qin Y, Qin J, Li B, Xu X, Geng Q, Jiang W, et al: SLIT2/ROBO1-miR-2181-RET/PLAG1: a new disease pathway involved in Hirschsprung's disease.J Cell Mol Med 2015, 19:1197-1207.

14. Sun XL, Wang ZL, Wu Q, Jin SQ, Yao J, Cheng H: LncRNA RMST activates TAK1-mediated NF-kappaB signaling and promotes activation of microglial cells via competitively binding with hnRNPK.IUBMB Life 2019, 71:1785-1793.

15. Collignon J, Sockanathan S, Hacker A, Cohen-Tannoudji M, Norris D, Rastan S, Stevanovic M, Goodfellow PN, Lovell-Badge R: A comparison of the properties of Sox-3 with Sry and two related genes, Sox-1 and Sox-2.Development 1996, 122:509-520.

16. Schepers GE, Teasdale RD, Koopman P: Twenty pairs of sox: extent, homology, and nomenclature of the mouse and human sox transcription factor gene families.Dev Cell 2002, 3:167-170.

17. Liu K, Xie F, Gao A, Zhang R, Zhang L, Xiao Z, Hu Q, Huang W, Huang Q, Lin B, et al: SoX2 regulates multiple malignant processes of breast cancer development through the SOX2/miR-181a-5p, miR30e-5p/TUSC3 axis. Mol Cancer 2017, 16:62.

18. Alqadah A, Hsieh YW, Vidal B, Chang C, Hobert O, Chuang CF: Postmitotic diversification of olfactory neuron types is mediated by differential activities of the HMG-box transcription factor SOX-2. EMBO J 2015, 34:2574-2589.

19. Schaefer T, Lengerke C: SOX2 protein biochemistry in stemness, reprogramming, and cancer: the $\mathrm{PI3K} / \mathrm{AKT} / \mathrm{SOX} 2$ axis and beyond.Oncogene 2020, 39:278-292.

20. Sannino G, Marchetto A, Ranft A, Jabar S, Zacherl C, Alba-Rubio R, Stein S, Wehweck FS, Kiran MM, Holting TLB, et al: Gene expression and immunohistochemical analyses identify SOX2 as major risk factor for overall survival and relapse in Ewing sarcoma patients.EBioMedicine 2019, 47:156-162.

21. Ng SY, Bogu GK, Soh BS, Stanton LW: The long noncoding RNA RMST interacts with SOX2 to regulate neurogenesis. Mol Cell 2013, 51:349-359. 
22. Moradimotlagh A, Arefian E, Rezazadeh Valojerdi R, Ghaemi S, Jamshidi Adegani F, Soleimani M: MicroRNA-129 Inhibits Glioma Cell Growth by Targeting CDK4, CDK6, and MDM2.Mol Ther Nucleic Acids 2019, 19:759-764.

23. Pan J, Fang S, Tian H, Zhou C, Zhao X, Tian H, He J, Shen W, Meng X, Jin X, Gong Z: IncRNA $\mathrm{JPX} / \mathrm{miR}-33 \mathrm{a}-5 \mathrm{p} /$ Twist 1 axis regulates tumorigenesis and metastasis of lung cancer by activating Wnt/beta-catenin signaling.Mol Cancer 2020, 19:9.

24. Tai F, Gong K, Song K, He Y, Shi J: Enhanced JunD/RSK3 signalling due to loss of BRD4/FOXD3/miR548d-3p axis determines BET inhibition resistance. Nat Commun 2020, 11:258.

25. Gunadi, Budi NYP, Kalim AS, Santiko W, Musthofa FD, Iskandar K, Makhmudi A: Aberrant expressions of miRNA-206 target, FN1, in multifactorial Hirschsprung disease. Orphanet J Rare Dis 2019, 14:5.

26. Zhi Z, Zhu H, Lv X, Lu C, Li Y, Wu F, Zhou L, Li H, Tang W: IGF2-derived miR-483-3p associated with Hirschsprung's disease by targeting FHL1.J Cell Mol Med 2018, 22:4913-4921.

27. Hui L, Wu H, Yang N, Guo X, Jang X: Identification of prognostic microRNA candidates for head and neck squamous cell carcinoma. Oncol Rep 2016, 35:3321-3330.

28. Southard-Smith EM, Kos L, Pavan WJ: Sox10 mutation disrupts neural crest development in Dom Hirschsprung mouse model.Nat Genet 1998, 18:60-64.

29. Wakamatsu Y, Endo Y, Osumi N, Weston JA: Multiple roles of Sox2, an HMG-box transcription factor in avian neural crest development.Dev Dyn 2004, 229:74-86.

30. lida H, Furukawa Y, Teramoto M, Suzuki H, Takemoto T, Uchikawa M, Kondoh H: Sox2 gene regulation via the $\mathrm{D} 1$ enhancer in embryonic neural tube and neural crest by the combined action of SOX2 and ZIC2.Genes Cells 2020, 25:242-256.

31. Cimadamore F, Fishwick K, Giusto E, Gnedeva K, Cattarossi G, Miller A, Pluchino S, Brill LM, BronnerFraser M, Terskikh AV: Human ESC-derived neural crest model reveals a key role for SOX2 in sensory neurogenesis. Cell Stem Cell 2011, 8:538-551.

32. Amador-Arjona A, Cimadamore F, Huang CT, Wright R, Lewis S, Gage FH, Terskikh AV: SOX2 primes the epigenetic landscape in neural precursors enabling proper gene activation during hippocampal neurogenesis. Proc Natl Acad Sci U S A 2015, 112:E1936-1945.

33. Wang Y, Zhou J, Wang Z, Wang P, Li S: Upregulation of SOX2 activated LncRNA PVT1 expression promotes breast cancer cell growth and invasion. Biochem Biophys Res Commun 2017, 493:429-436.

34. Wang Z, Yang B, Zhang M, Guo W, Wu Z, Wang Y, Jia L, Li S, Cancer Genome Atlas Research N, Xie W, Yang D: IncRNA Epigenetic Landscape Analysis Identifies EPIC1 as an Oncogenic IncRNA that Interacts with MYC and Promotes Cell-Cycle Progression in Cancer.Cancer Cell 2018, 33:706-720 e709.

35. Su Y, Wen Z, Shen Q, Zhang H, Peng L, Chen G, Zhu Z, Du C, Xie H, Li H, et al: Long non-coding RNA LOC100507600 functions as a competitive endogenous RNA to regulate BMI1 expression by sponging miR128-1-3p in Hirschsprung's disease. Cell Cycle 2018, 17:459-467.

36. Li Y, Zhou L, Lu C, Shen Q, Su Y, Zhi Z, Wu F, Zhang H, Wen Z, Chen G, et al: Long non-coding RNA FAL1 functions as a ceRNA to antagonize the effect of miR-637 on the down-regulation of AKT1 in 
Hirschsprung's disease. Cell Prolif 2018, 51:e12489.

37. Chopra N, Wang R, Maloney B, Nho K, Beck JS, Pourshafie N, Niculescu A, Saykin AJ, Rinaldi C, Counts SE, Lahiri DK: MicroRNA-298 reduces levels of human amyloid-beta precursor protein (APP), beta-site APP-converting enzyme 1 (BACE1) and specific tau protein moieties.Mol Psychiatry 2020.

38. Lee IH, Sohn M, Lim HJ, Yoon S, Oh H, Shin S, Shin JH, Oh SH, Kim J, Lee DK, et al: Ahnak functions as a tumor suppressor via modulation of TGFbeta/Smad signaling pathway.Oncogene 2014, 33:4675-4684.

39. Chen B, Wang J, Dai D, Zhou Q, Guo X, Tian Z, Huang X, Yang L, Tang H, Xie X: AHNAK suppresses tumour proliferation and invasion by targeting multiple pathways in triple-negative breast cancer. $J$ Exp Clin Cancer Res 2017, 36:65.

\section{Tables}

Table 1. Primer Sequences for Quantitative RT-PCR

\begin{tabular}{|c|c|}
\hline Target Gene & Primer Sequence (5' to $\left.3^{\prime}\right)$ \\
\hline \multirow[t]{2}{*}{ GAPDH } & F: GCACCGTCAAGGCTGAGAAC \\
\hline & R: GGATCTCGCTCCTGGAAGATG \\
\hline \multirow[t]{2}{*}{ RMST } & F: ACTTCTGAGTGGTATGCTGCT \\
\hline & R: GGATGGTGGTTTTGATGTTTC \\
\hline \multirow[t]{2}{*}{ sox2 } & F: TTGCTGCCTCTTTAAGACTAGGA \\
\hline & R: CTGGGGCTCAAACTTCTCTC \\
\hline \multirow[t]{2}{*}{ AHNAK } & F: TACCCTTCCTAAGGCTGACATT \\
\hline & R: TTGGACCCTTGAGTTTTGCAT \\
\hline \multirow[t]{2}{*}{ miR-1251 } & F: ACACTCCAGCTGGG ACTCTAGCTGCCAAA \\
\hline & R: CTCAACTGGTGTCGTGGAGTCGGCAATTCAGTTGAG AGCGCCTT \\
\hline \multirow[t]{2}{*}{ promoter region of miR-1251 } & F: TGGACAAGCTGAAGATATGGACA \\
\hline & R: TGACCTCGATGGCAGTGATG \\
\hline
\end{tabular}

\section{Figures}


A

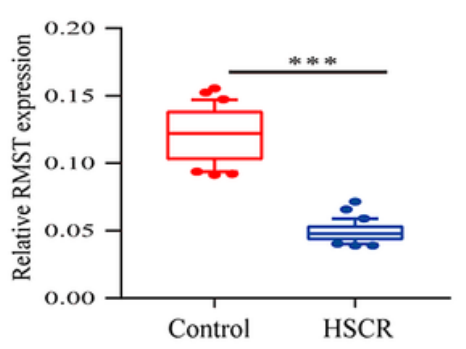

C

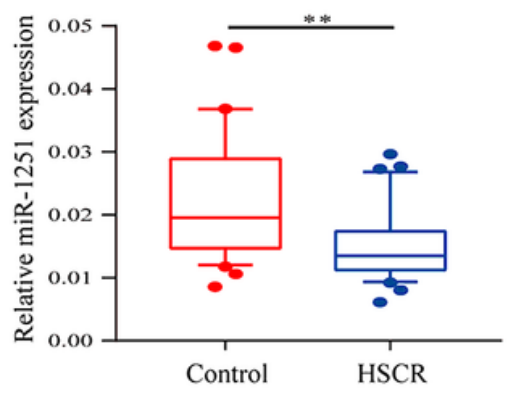

D
B

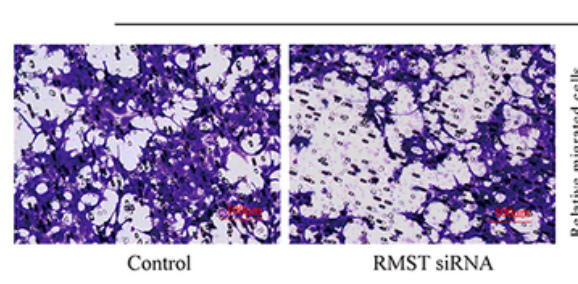

$293 \mathrm{~T}$
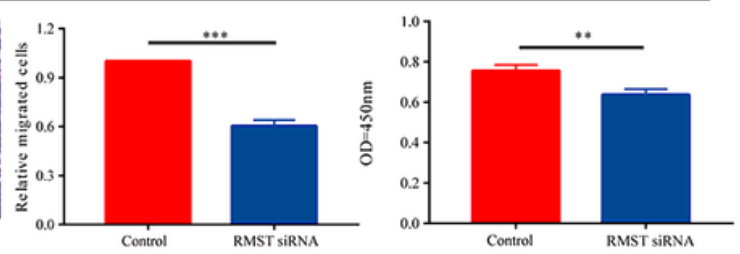

SH-SY5Y

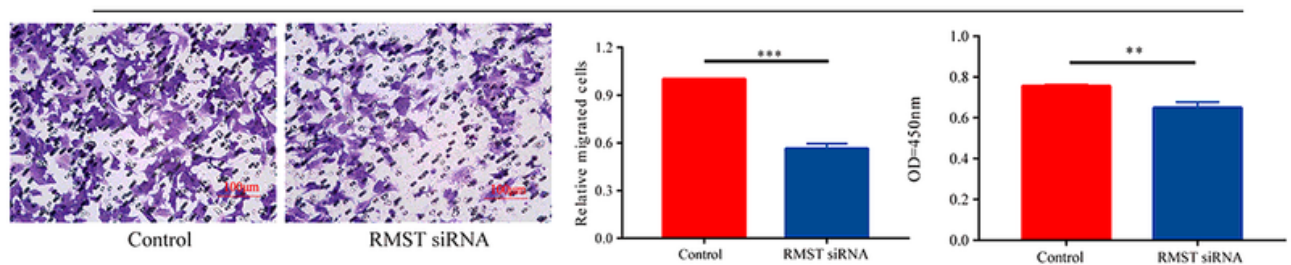

293T
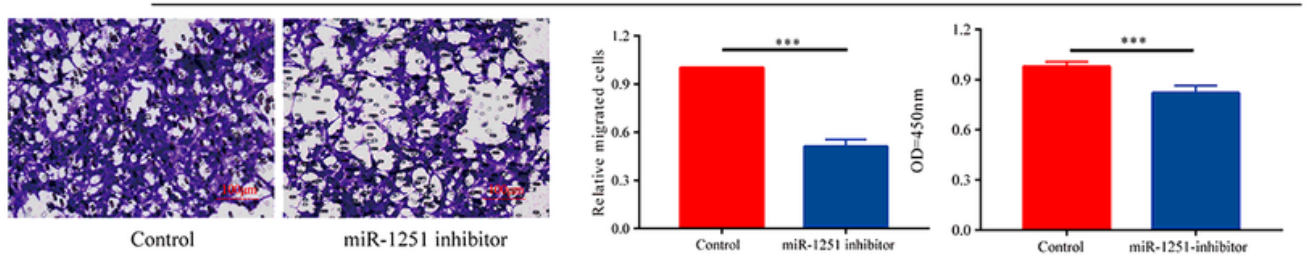

SH-SY5Y
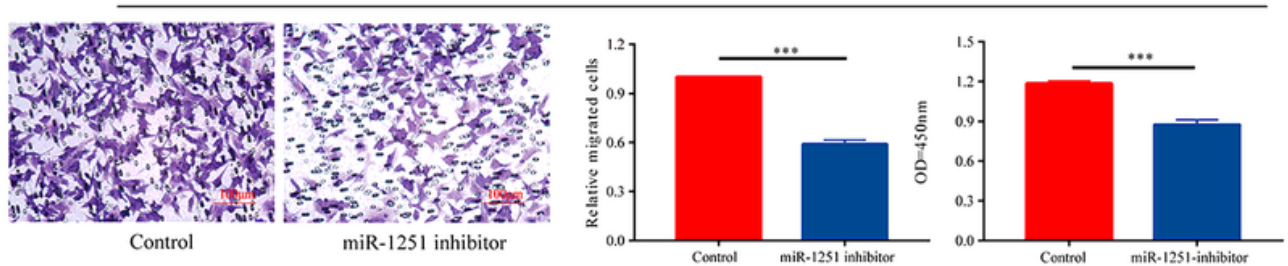

\section{Figure 1}

Down-regulation of RMST and miR-1251 in HSCR patients a The expression of RMST was downregulated obviously in stenosis tracts than control ones. b Transwell and CCK-8 assays showed cell migration and proliferation were attenuated when RMST was down-regulated. c The expression of miR1251 was down-regulated obviously in stenosis tracts than control ones. $\mathrm{d}$ Cell proliferation and migration were inhibited obviously when transfected with miR-1251 inhibitor. ${ }^{\star} * \mathrm{P}<0.01,{ }^{\star} * \star \mathrm{P}<0.001$ 
A

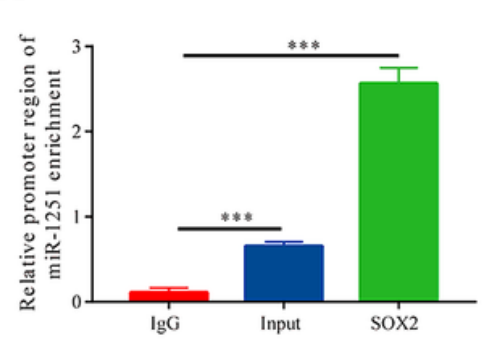

D

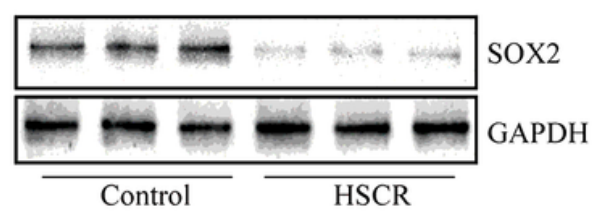

F

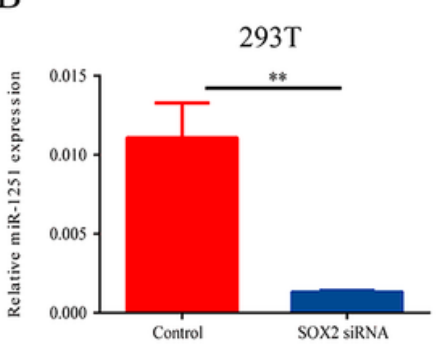

E
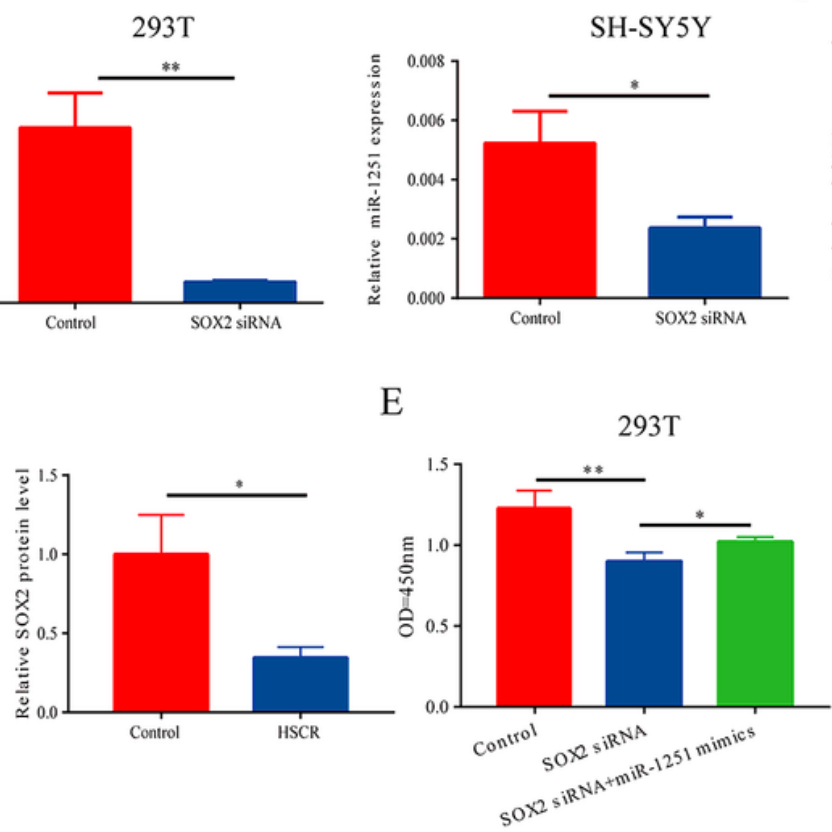

C

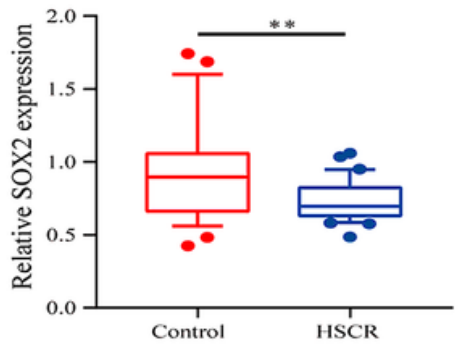

SH-SY5Y

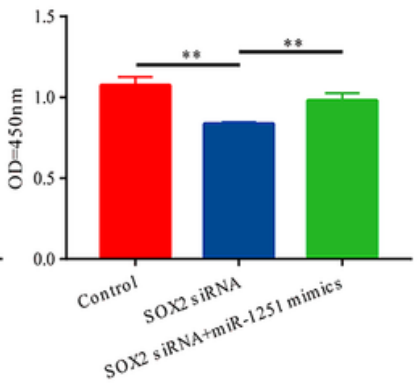

293T

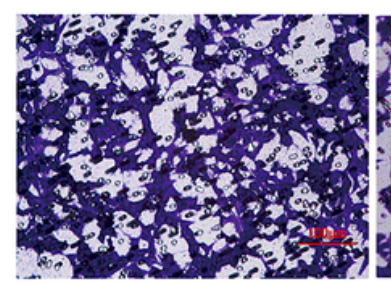

Control

G

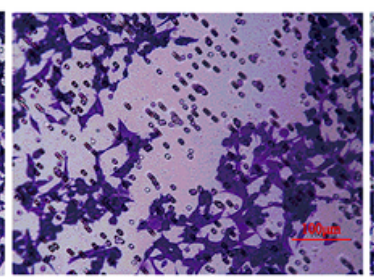

SOX2 siRNA

SOX2 siRNA+miR-1251 mimics

SH-SY5Y
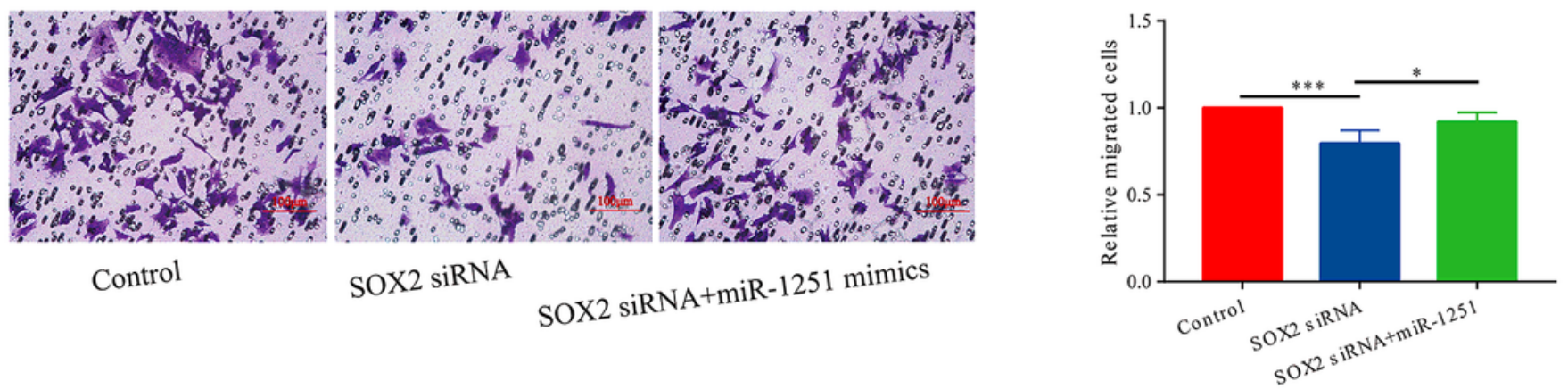

Figure 2

miR-1251 was transcriptionally regulated by SOX2 a CHIP assay showed SOX2 could bind to the promoter region of miR-1251. b After down regulating the expression of SOX2, the expression of miR1251 was lower than control group. c, $d$ The mRNA and protein expression of SOX2 in HSCR patients. e-g When transfected with si-SOX2, cell migration and proliferation were inhibited and the up-regulation of miR-1251 could partly reversed it. ${ }^{*} P<0.05,{ }^{*} * P<0.01,{ }^{*} * * P<0.001$ 
A

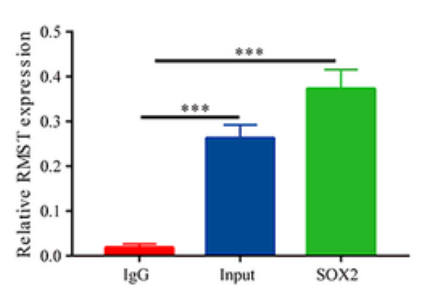

B

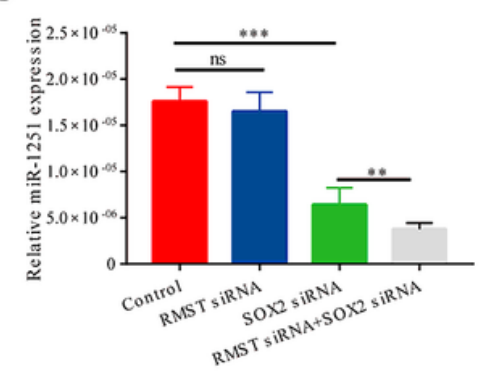

$\mathrm{D}$

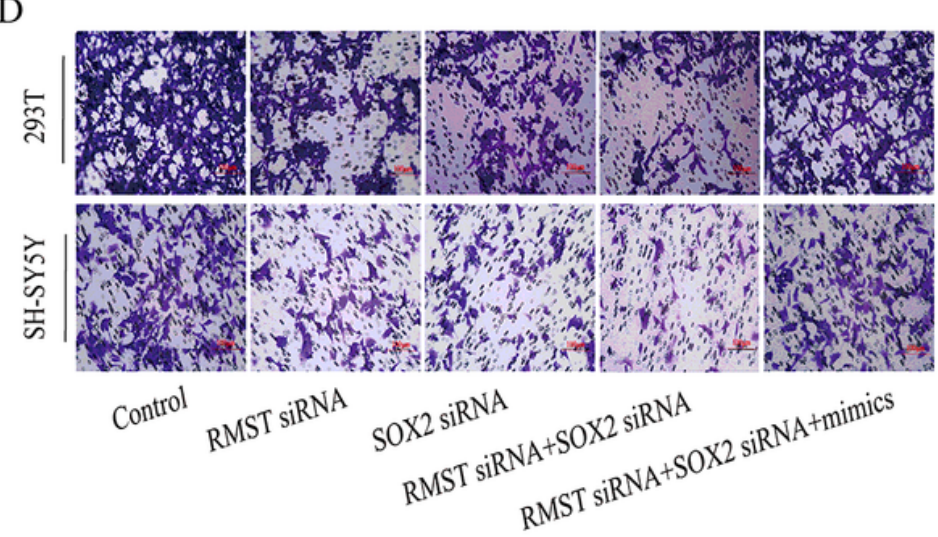

$\mathrm{C}$

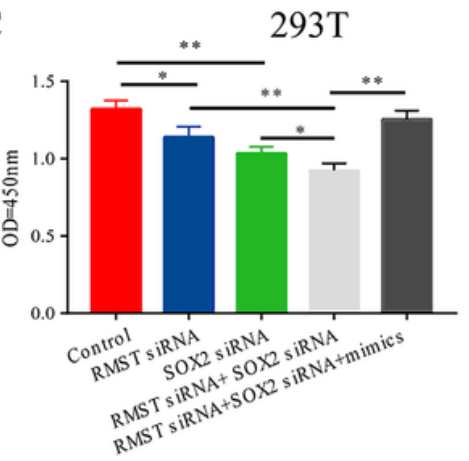

$293 \mathrm{~T}$

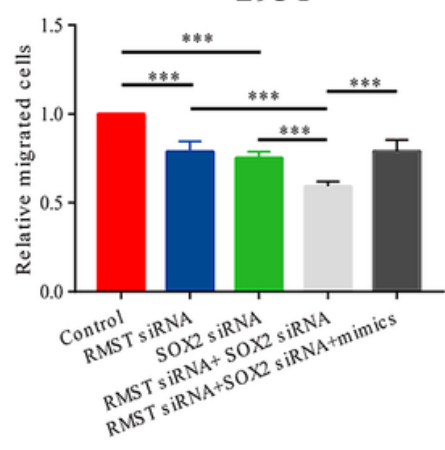

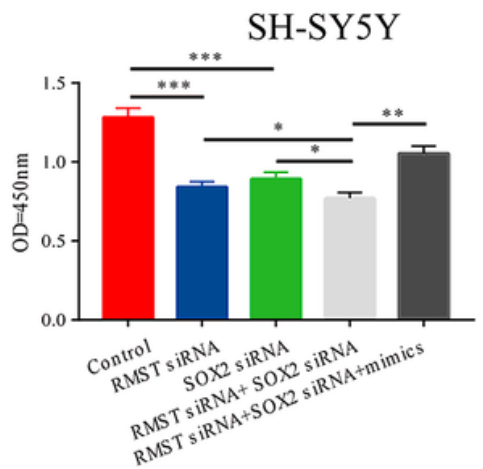

SH-SY5Y

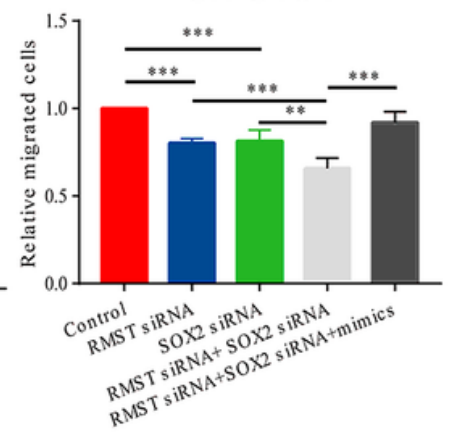

Figure 3

RMST functioned as a co-regulator of SOX2 a RIP assay showed RMST could bind to SOX2. b RMST functioned as a co-regulator of SOX2 and promoted the regulation effect of SOX2 on miR-1251. c, d CCK8 and Tranwell assays revealed that when the expression of RMST and SOX2 were both knockdown, the cell proliferation and migration were more weakened than just down-regulated RMST or SOX2 alone. $\star P<0.05, * \star P<0.01, * \star * P<0.001$ 
A

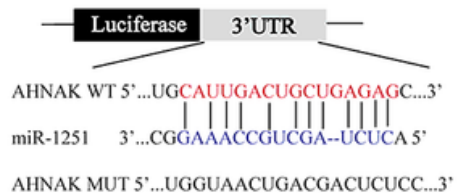

D Control $_{\text {miR-1251 inhibitor }}$ AHNAK

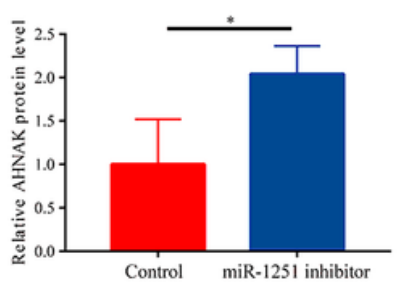

$\mathrm{H}$

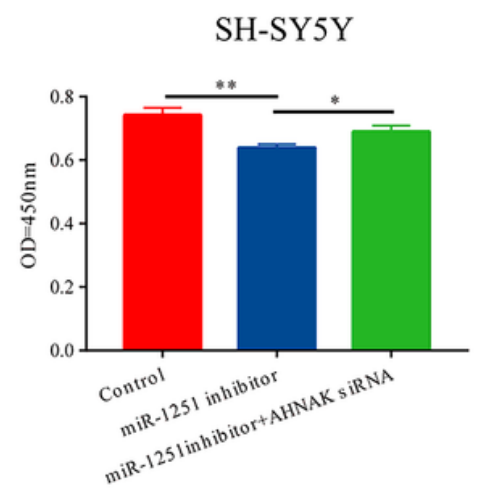

B
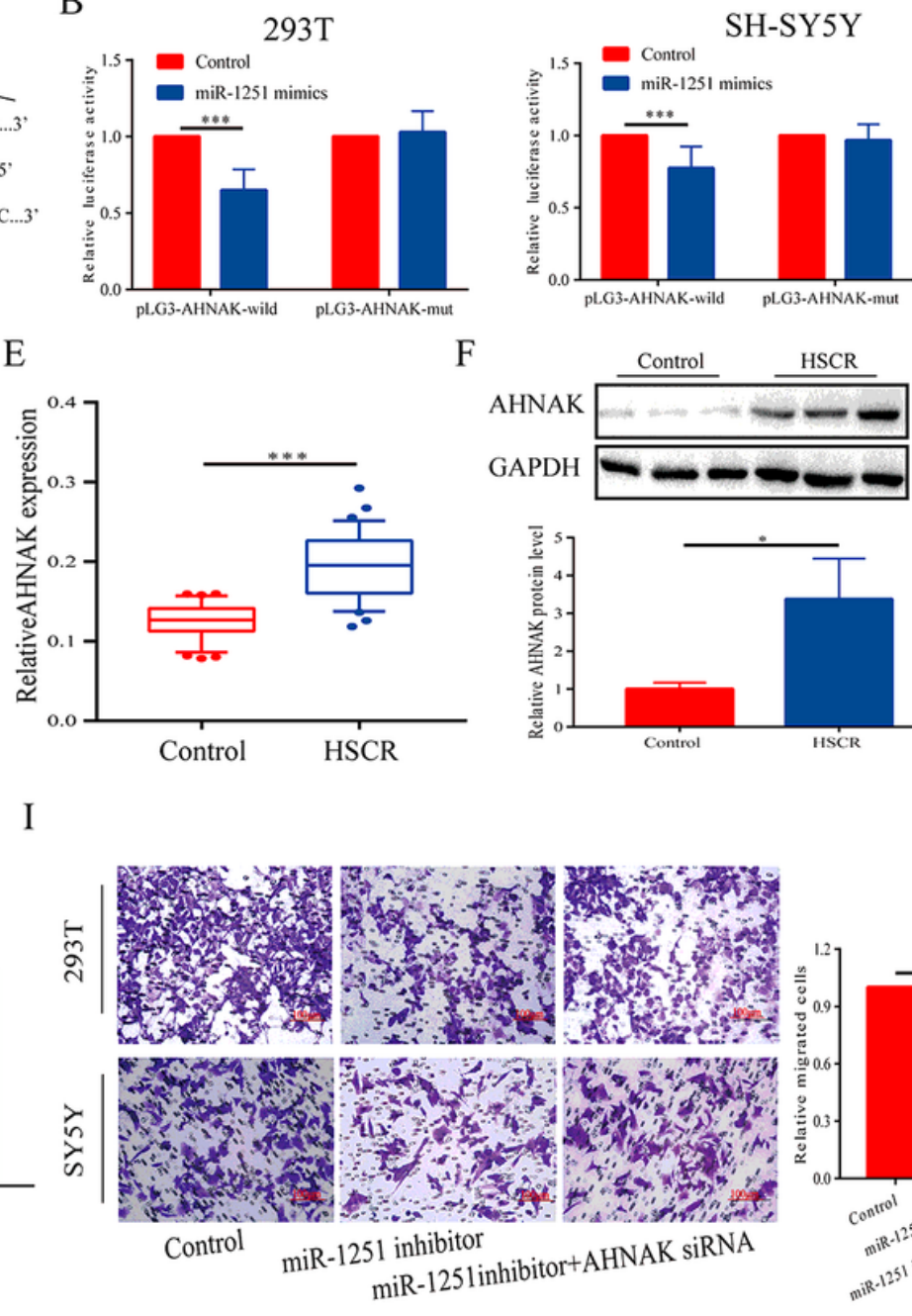

SH-SY5Y

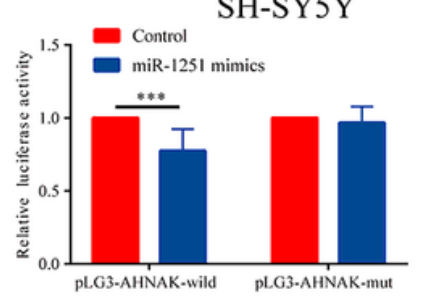

G
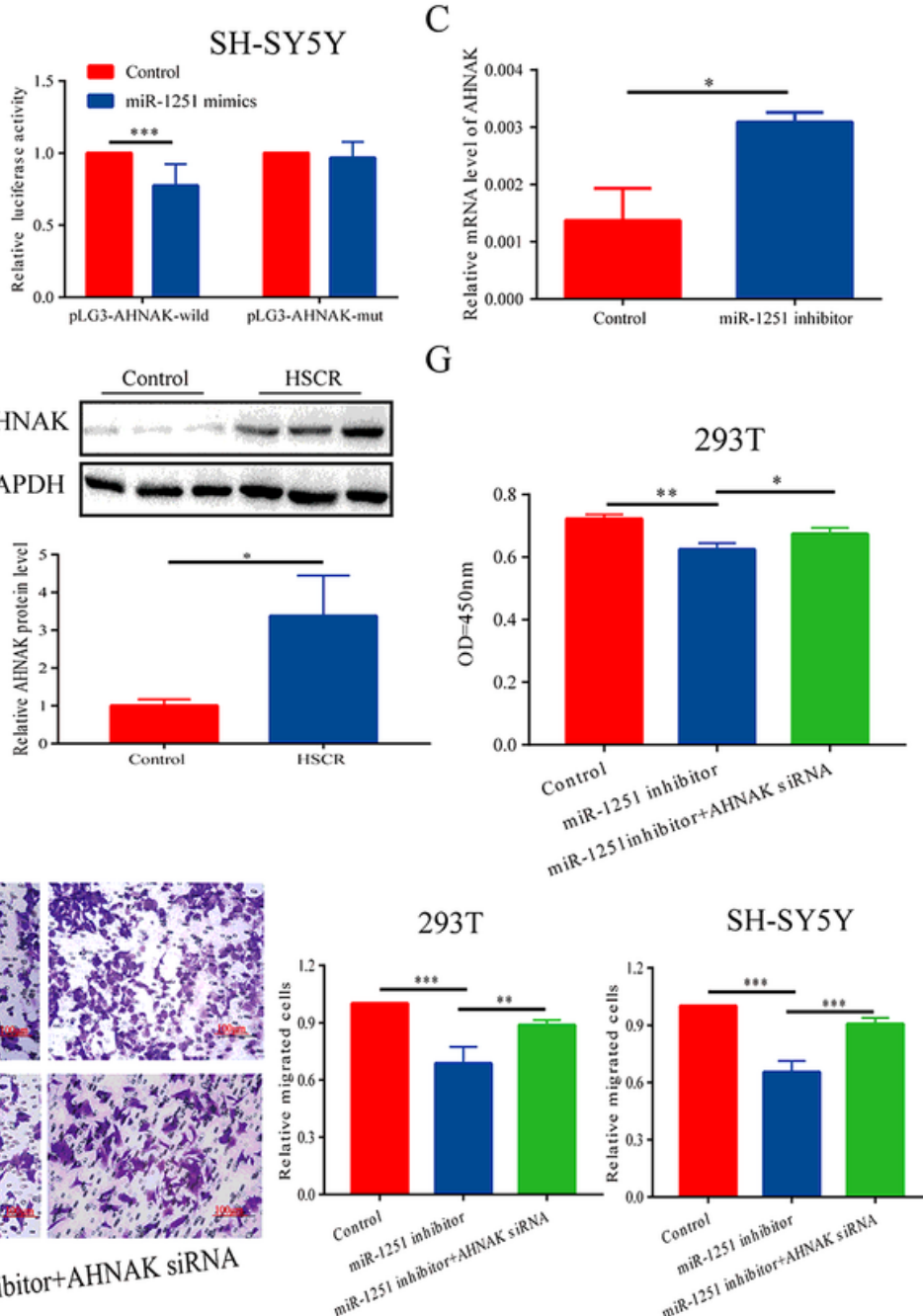

SH-SY5Y

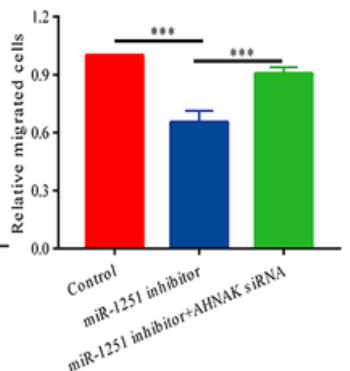

Figure 4

AHNAK was the target gene of miR-1251. a miR-1251 and AHNAK has potential binding sites. b Dulluciferase reporter gene assay confirmed the binding relationship between miR-1251 and AHNAK in 293T and SY5Y cells. c, d When miR-1251 was knocked down, the mRNA and protein level of AHNAK was upregulated, e, f The AHNAK mRNA and protein expression level in stenosis tracts was higher than control tracts. g-i The down-regulation of AHNAK could partly reverse the influence of miR-1251 inhibitor on cell migration and proliferation. ${ }^{*} \mathrm{P}<0.05,{ }^{*} \mathrm{P}<0.01,{ }^{*} * \mathrm{P}<0.001$ 
A

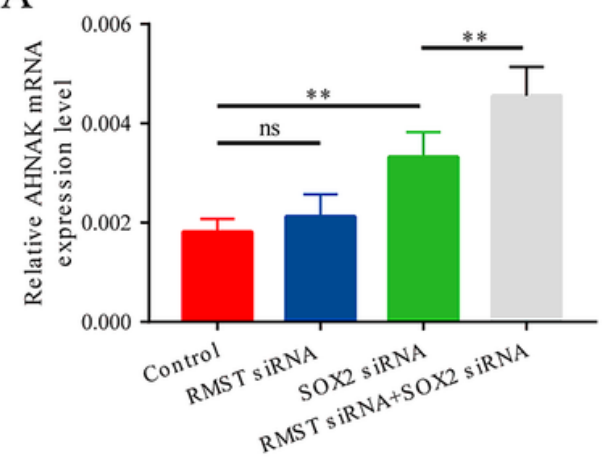

C
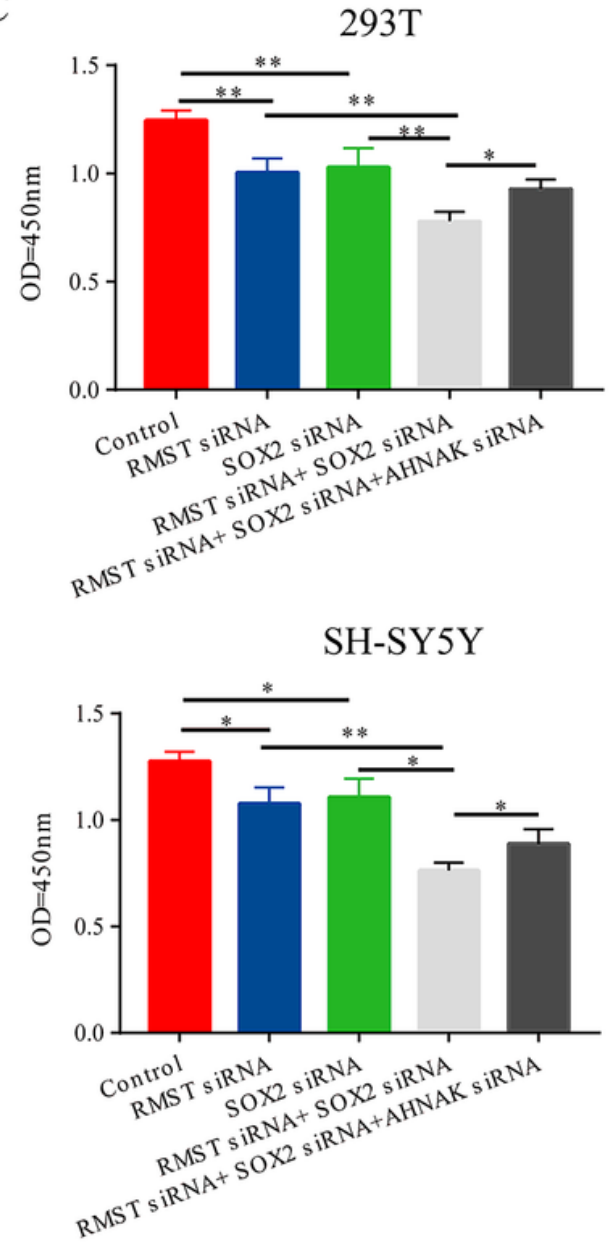

B

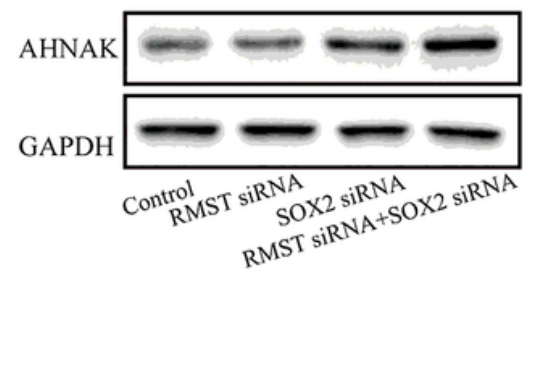

D

$293 \mathrm{~T}$
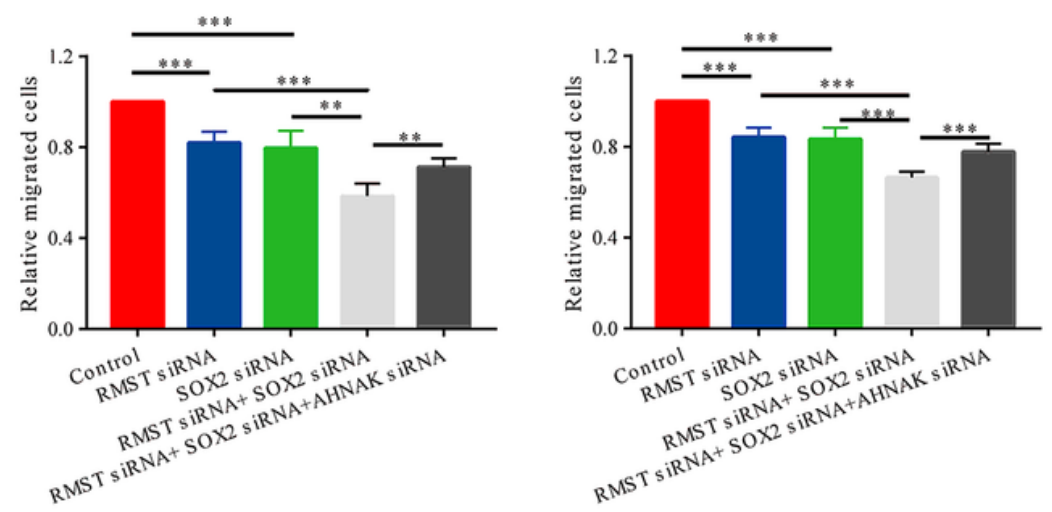

SH-SY5Y

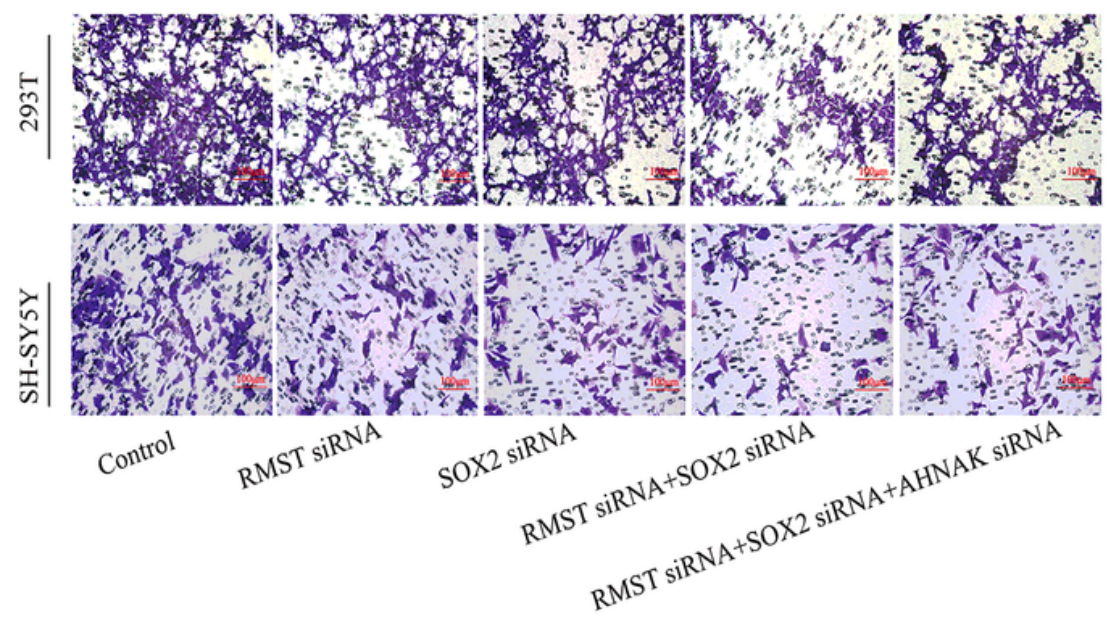

Figure 5

RMST played as a SOX2 transcription co-regulator to inhibit miR-1251 and raise AHNAK expression a, $b$ The mRNA and protein level of AHNAK in every group. c, d CCK-8 and Transwell assays showed that the combined inhibition of both RMST and SOX2 low expression on cell proliferation and migration could be partially alleviated by simultaneously downregulating the expression of AHNAK. ns $P \geq 0.05, * P<0.05$, $\star \star P<0.01, * \star * P<0.001$

\section{Supplementary Files}


This is a list of supplementary files associated with this preprint. Click to download.

- SupplementFigure.tif 